Supporting Information

\title{
Passivating Quantum Dot Carrier Transport Layer with Metal Salts
}

Zihan Chen, ${ }^{1,2}$ Yaohong Zhang, ${ }^{3}$ Zhi Li Teh, ${ }^{2}$ Jianfeng Yang, ${ }^{2}$ Lin Yuan, ${ }^{4}$ Gavin J. Conibeer, ${ }^{2}$

Robert J.Patterson, ${ }^{2}$ Qing Shen, ${ }^{3}$ Shujuan Huang, ${ }^{* 4}$ Zhilong Zhang*1

${ }^{1}$ Cavendish Laboratory

University of Cambridge

Cambridge CB3 OHE, United Kingdom

${ }^{2}$ Australian Centre for Advanced Photovoltaics

School of Photovoltaic and Renewable Energy Engineering

University of New South Wales (UNSW)

Sydney 2052, NSW, Australia

${ }^{3}$ Faculty of Informatics and Engineering

The University of Electro-Communications

Tokyo 182-8585, Japan

${ }^{4}$ School of Engineering

Macquarie University Sustainable Energy Research Centre

Macquarie University

Sydney 2109, NSW, Australia

\section{Corresponding Authors}

\section{Shujuan Huang}

Email: shujuan.huang@mq.edu.au

\section{Zhilong Zhang}

Email: zz359@cam.ac.uk 


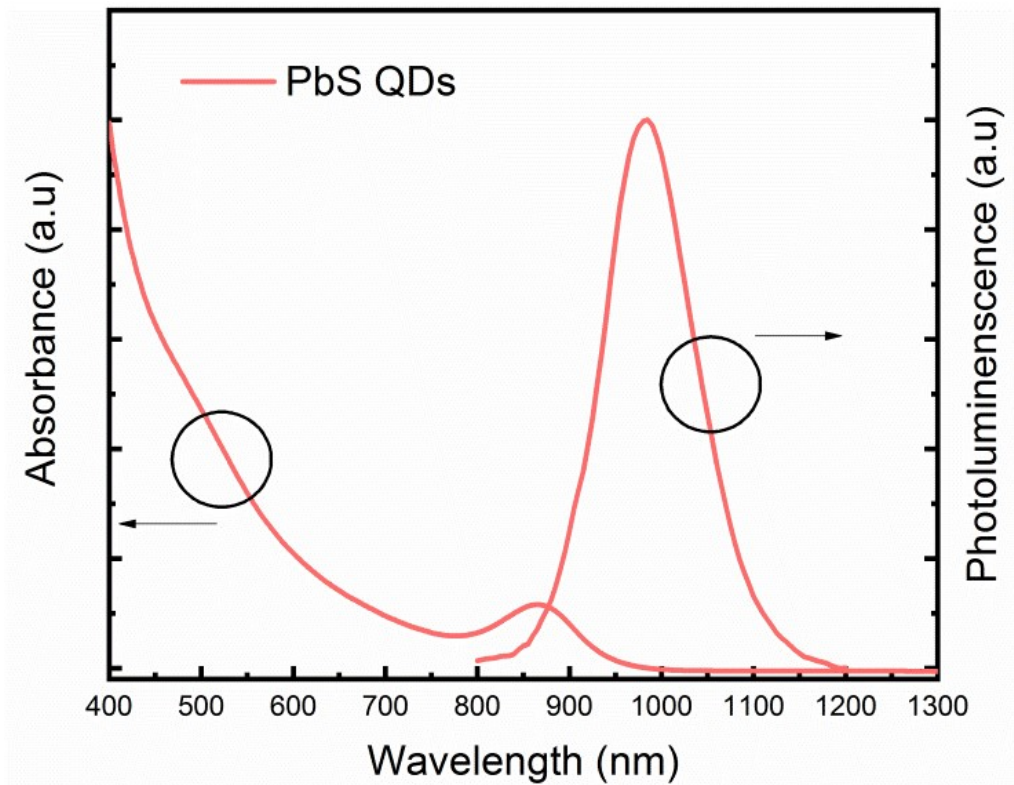

Figure S1. Absorption and photoluminescence spectra of the as-synthesized PbS QDs used in this work. 


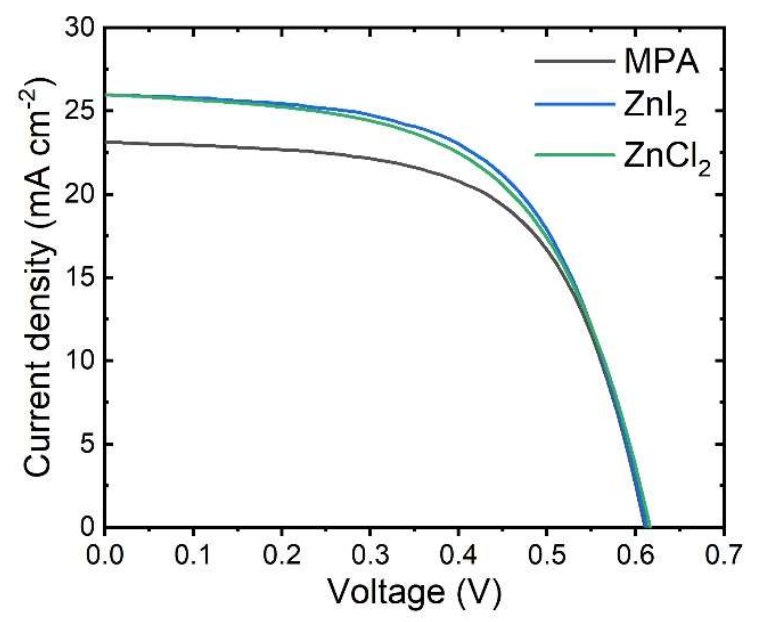

Figure S2. J-V curves of the device with zinc chloride $\left(\mathrm{ZnCl}_{2}\right)$ treated HTL. The device performance parameters are: $\mathrm{V}_{\mathrm{OC}}$ of $616 \mathrm{mV}, \mathrm{J}_{\mathrm{SC}}$ of $25.9 \mathrm{~mA} \mathrm{~cm}{ }^{-2}, \mathrm{FF}$ of $57.9 \%$ and overall PCE of 9.3\%. Compared with MPA, the device performance is improved, however to a level slightly lower than that of $\mathrm{ZnI}_{2}$. 

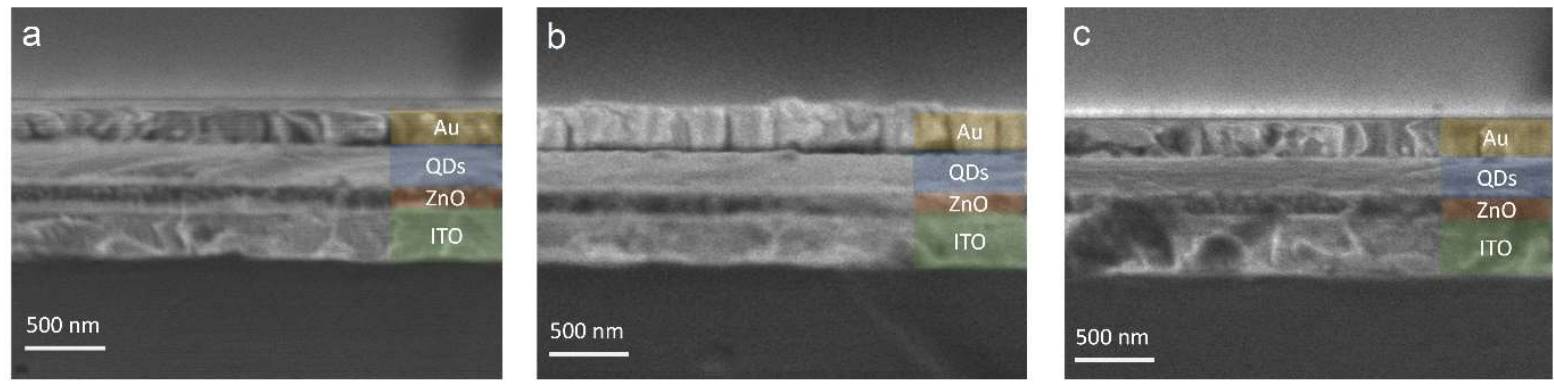

Figure S3. Cross-sectional SEM images of the (a) MPA, (b) ZnAc and (c) ZnI 2 devices. There is no obviously change in the cell structure. The film thicknesses of each layer are also similar.

\section{Improved EQE after zinc salt treatments:}

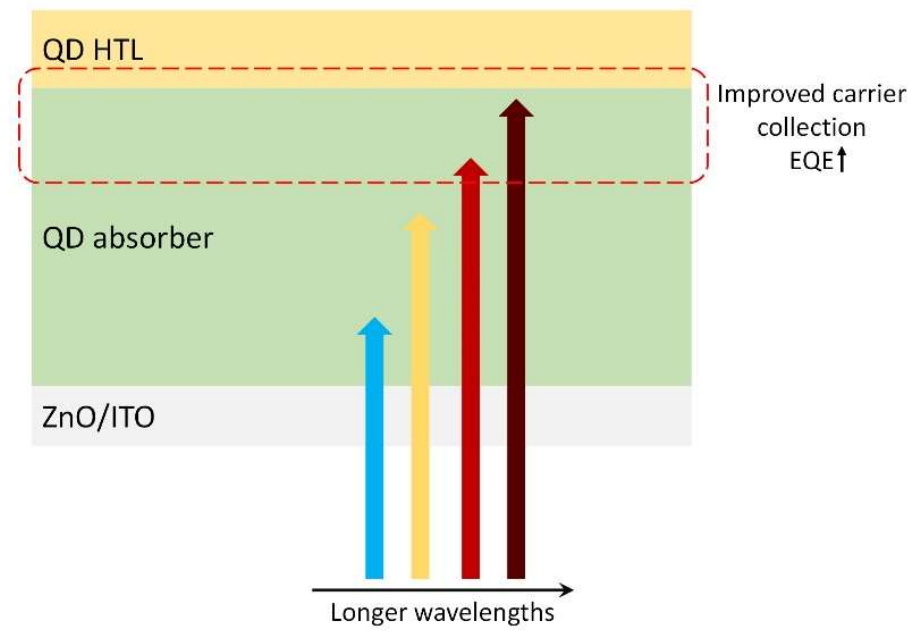

Figure S4. Schematic of photon penetration into the QD absorber. The incident photons are from the $\mathrm{ZnO} / \mathrm{ITO}$ side and those with longer wavelengths has longer penetration length into the absorber. This is due to the relatively low absorption of QDs in the region with longer wavelengths, as shown in Figure S2. In this case, the photons with longer wavelengths are more likely to be absorbed near the absorber/HTL interfaces. As the zinc salt treatments can reduce recombination and hence improve carrier collection near these interfaces, the EQE of the corresponding photons are also improved, as shown in Figure $2 \mathrm{~b}$ in the main text. 


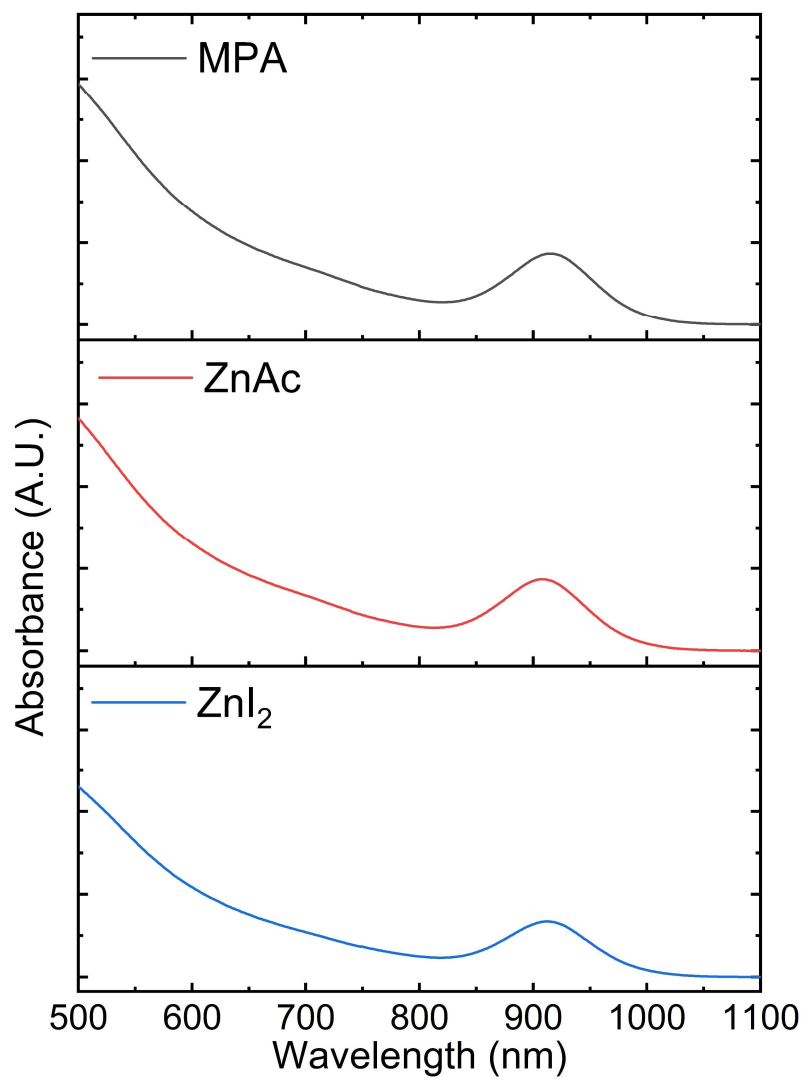

Figure S5. Steady-date absorption spectra of the HTLs with different treatments. 


\section{Spectro-temporal TA maps of the HTLs under higher fluences:}
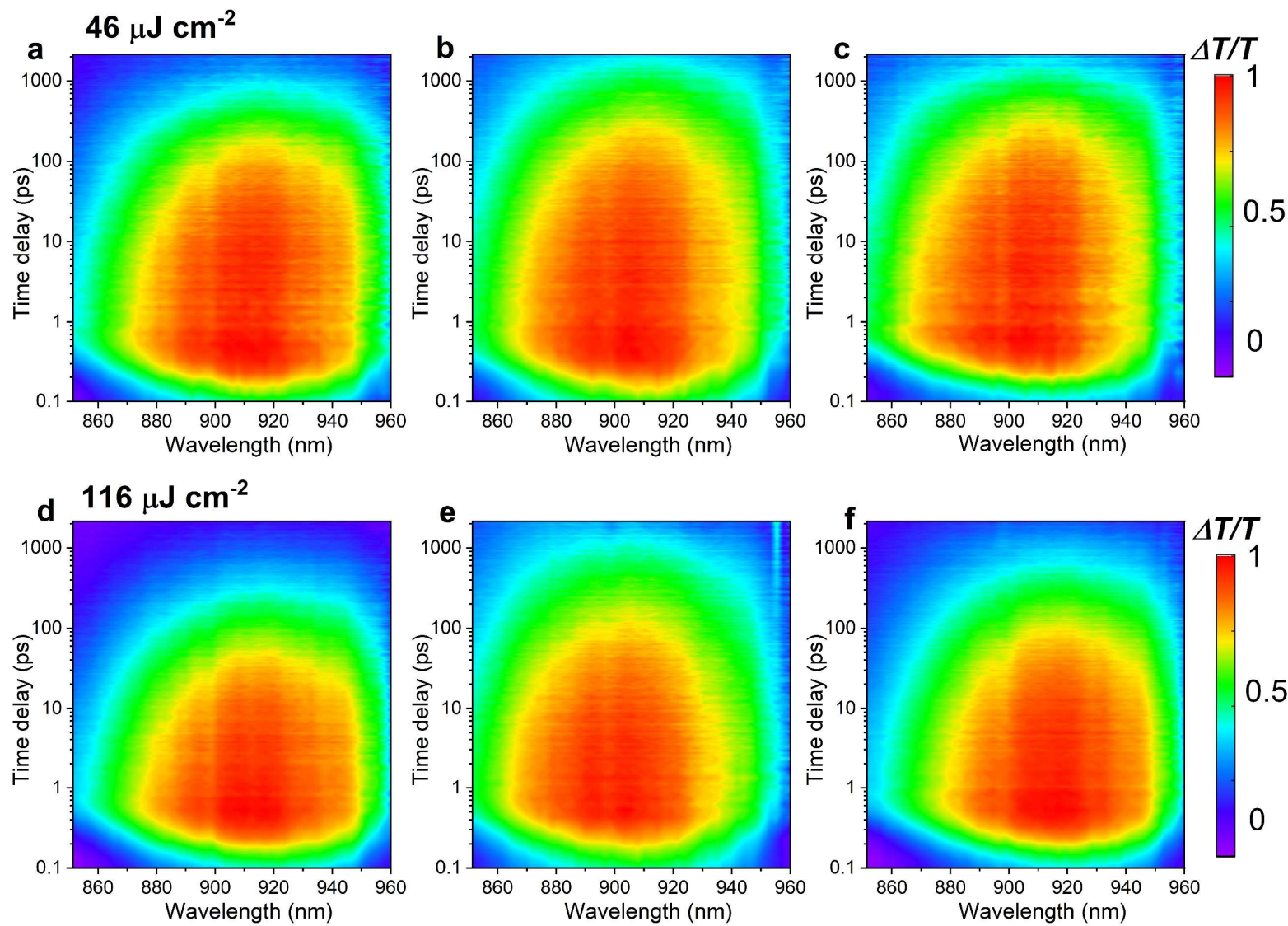

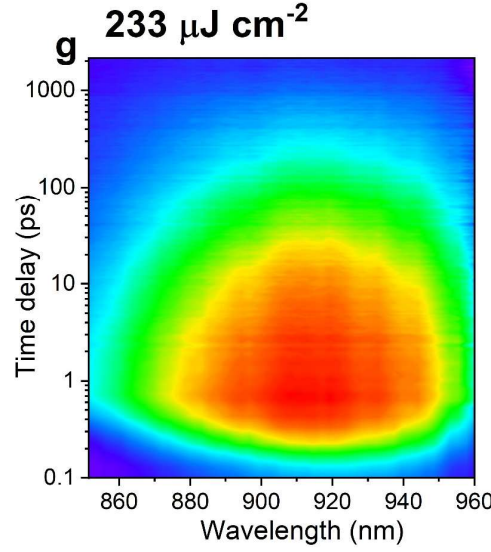

MPA

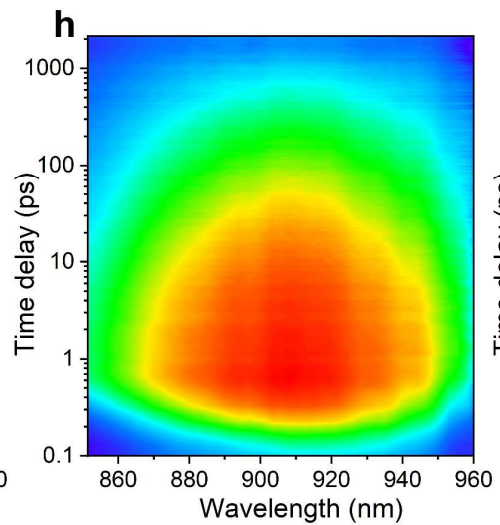

ZnAc

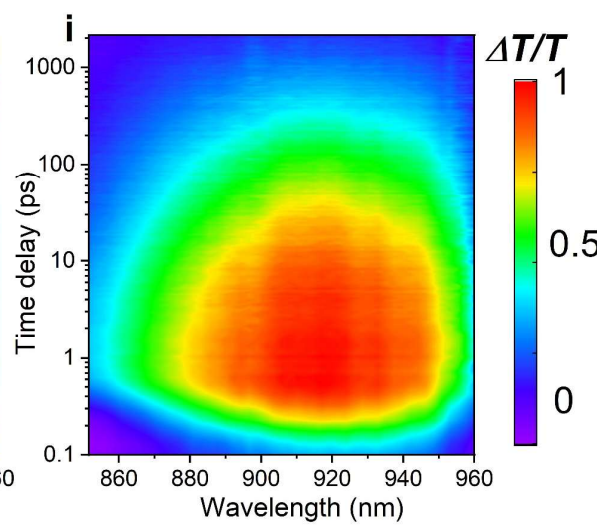

$\mathrm{ZnI}_{2}$

Figure S6. TA results of the HTLs under higher fluences than that shown in Figure 3 in the main text. a-c, $\mathbf{d - f}$ and $\mathbf{g - i}$ correspond to the results under the fluence of $46 \mu \mathrm{J} \mathrm{cm}^{-2}, 116 \mu \mathrm{J}$ $\mathrm{cm}^{-2}$ and $233 \mu \mathrm{J} \mathrm{cm}^{-2}$, respectively. Left to right: the pristine MPA HTL, ZnAc and $\mathrm{ZnI}_{2}$ 
treated HTLs, respectively. The spectral red-shifts and photo-bleach lifetime show the same trend as that presented in Figure 3. Kinetics fitted results are shown in Figure S5 and Table S1.

\section{TA signal decay kinetics under higher excitation fluences:}
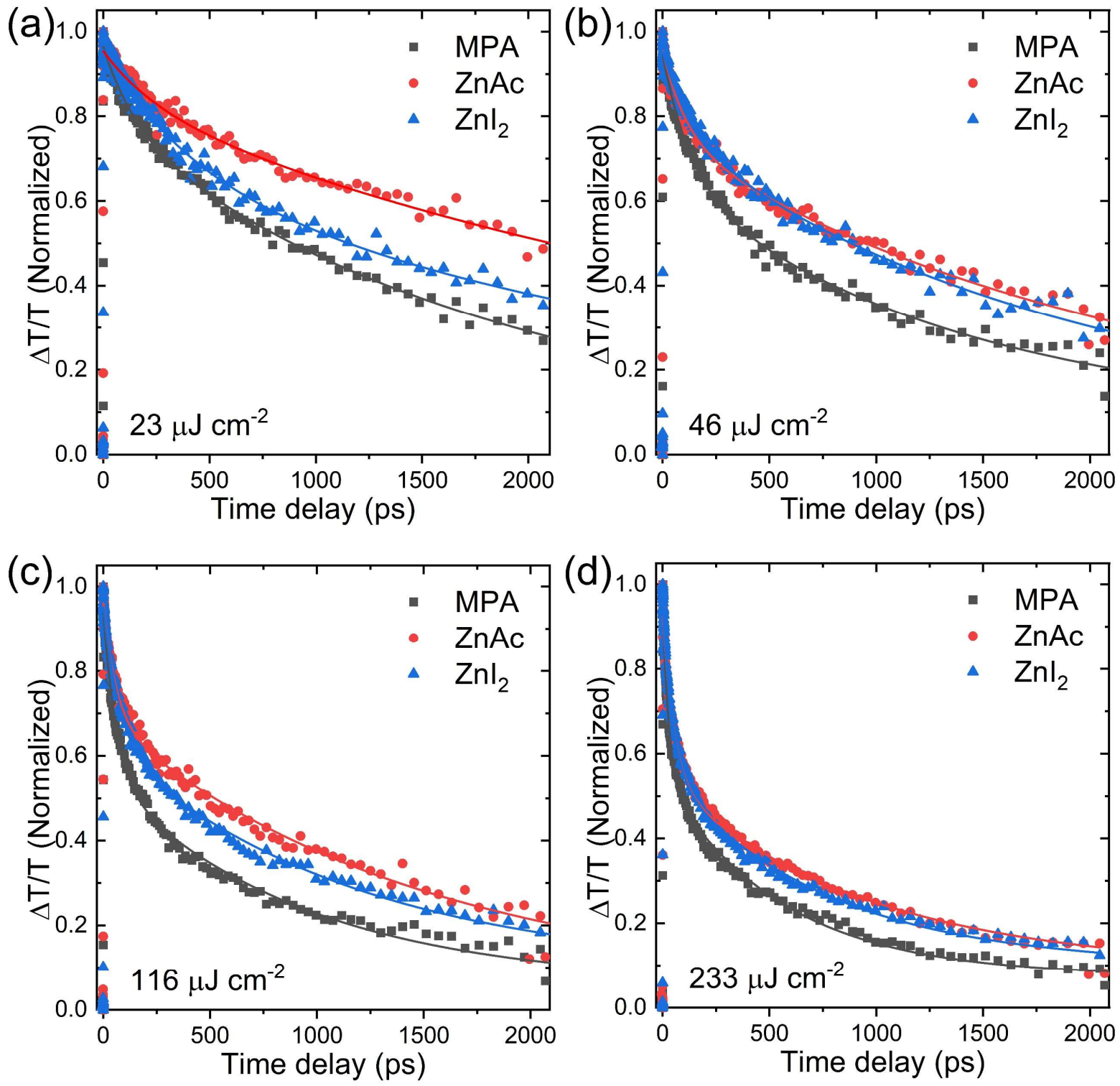

Figure S7. TA signal decay kinetics of the HTLs under different excitation energy intensity.

The measurements were performed under $520 \mathrm{~nm}$ laser with energy intensity of (a) $23 \mathrm{uJ} \mathrm{cm}^{-2}$ (presented in the main text), (b) $46 \mathrm{uJ} \mathrm{cm}^{-2}$, (c) $116 \mathrm{uJ} \mathrm{cm}^{-2}$ and (d) $233 \mathrm{uJ} \mathrm{cm}^{-2}$. 
The curves are fitted with a bi-exponential decay model, such that

$$
\mathrm{A}(\mathrm{t})=\alpha_{1} e^{-t / \tau_{1}}+\alpha_{2} e^{-t / \tau_{2}}
$$

The average lifetime is then calculated by the following equation and summarized in Table 2 in the main text.:

$$
\tau_{\text {avg }}=\left(a_{1} \times \tau_{1}+a_{2} \times \tau_{2}\right) /\left(a_{1}+a_{2}\right)
$$




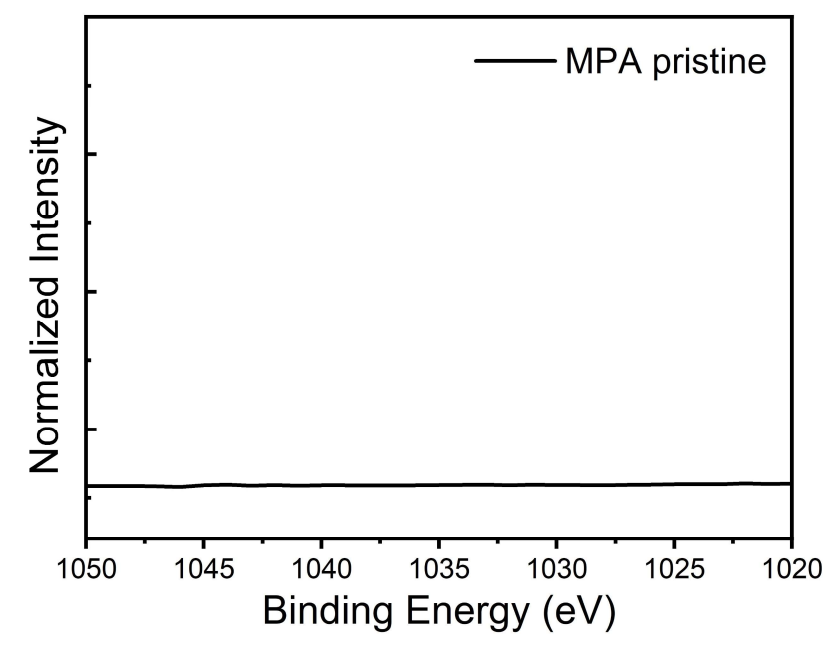

Figure S8. XPS results of the MPA pristine HTL in the region corresponding to $Z n \mathrm{p}_{1 / 2}$ $(1045 \mathrm{eV})$ and $\mathrm{Zn} 2 \mathrm{p}_{3 / 2}(1022 \mathrm{eV})$ peaks. Compared to those of the zinc salt treated HTLs as presented in the main text (Figure. 4a), there is no obvious features in this region, indicating the absence of $\mathrm{Zn}^{2+}$ in the MPA HTL or any other contents that have features overlapping with the $\mathrm{Zn} 2 \mathrm{p}$ peaks. 


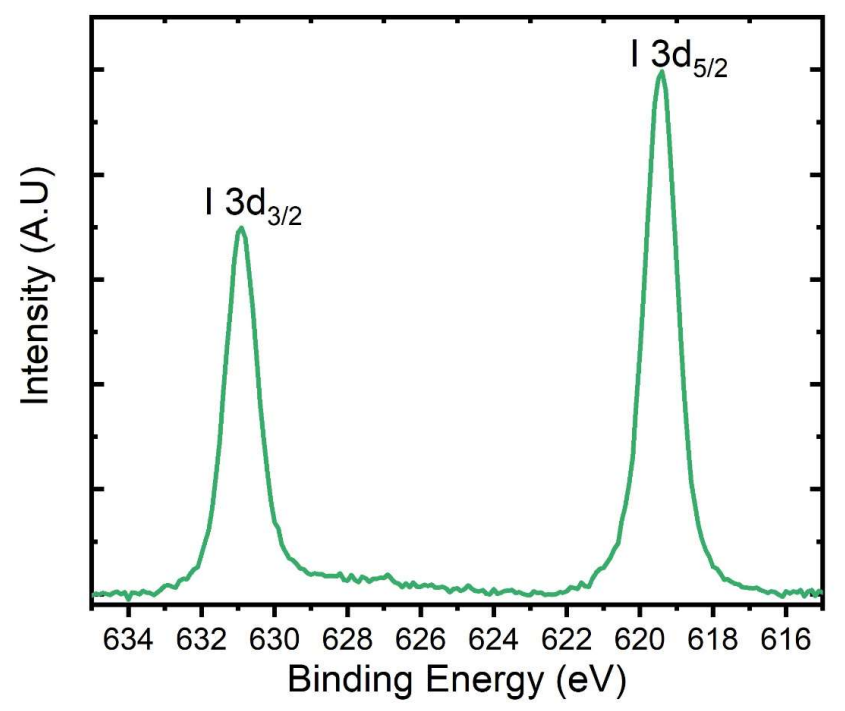

Figure S9. XPS spectra of the $\mathrm{ZnI}_{2}$ treated HTL, showing the I $3 d_{3 / 2}$ and $\mathrm{I} 3 \mathrm{~d}_{5 / 2}$ features, indicating incorporation of $\mathrm{I}^{-}$ligands into the HTL. 


\section{UPS spectra:}
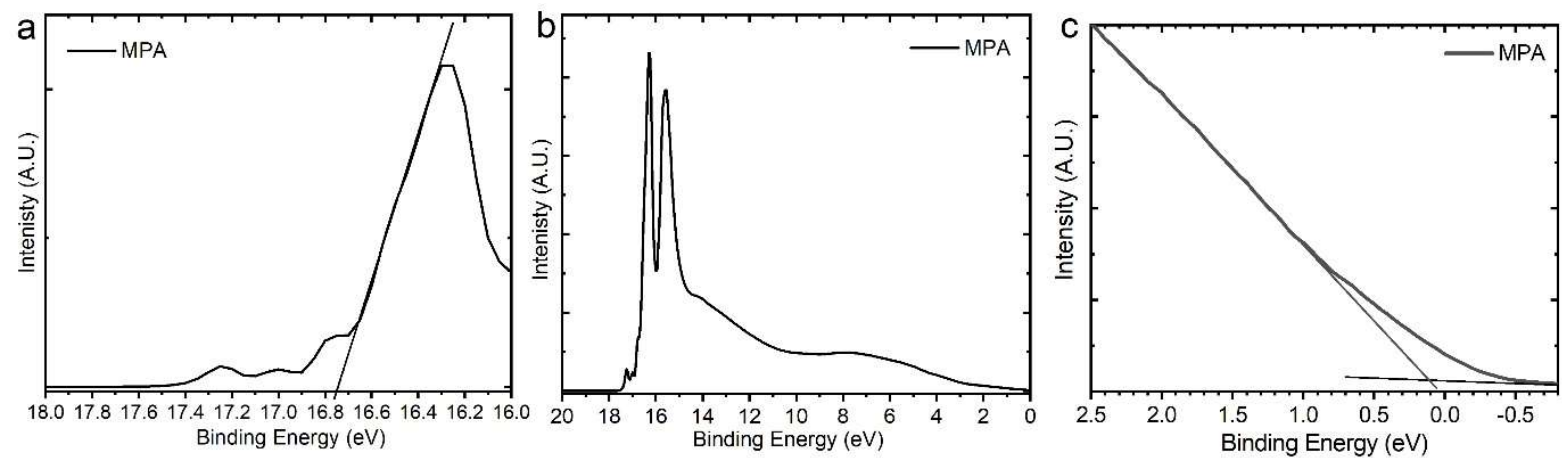

Figure S10. UPS results of the HTL with pure MPA (pristine reference). (a) High-bindingenergy region. (b) Full UPS spectra. (c) Low-binding-energy region.
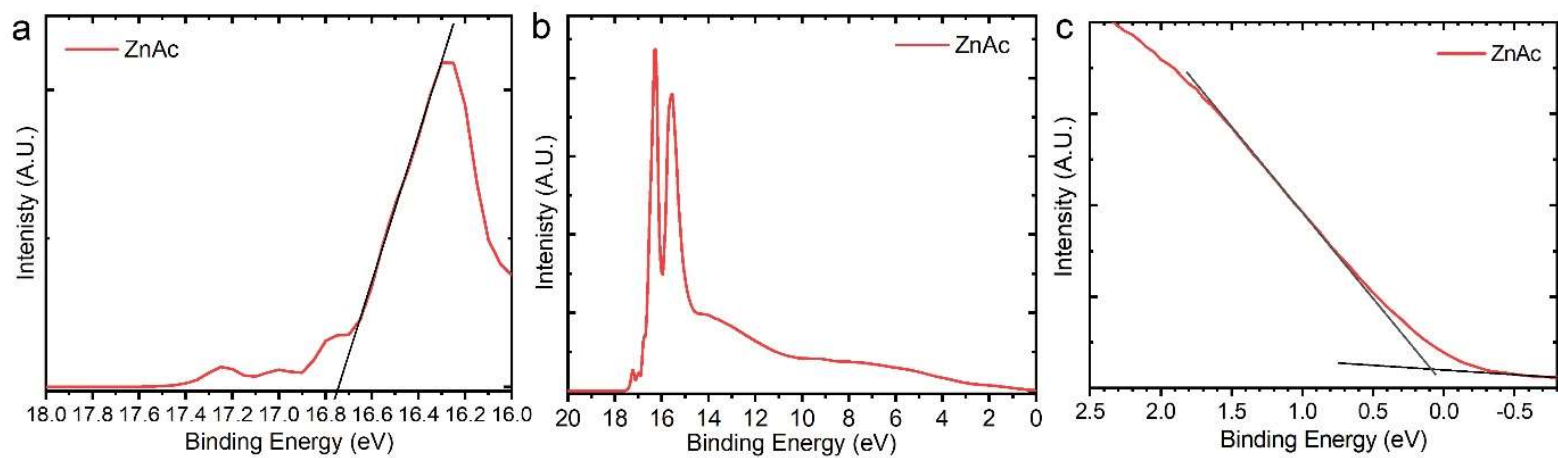

Figure S11. UPS results of the HTL with ZnAc treatment. (a) High-binding-energy region.

(b) Full UPS spectra. (c) Low-binding-energy region. 

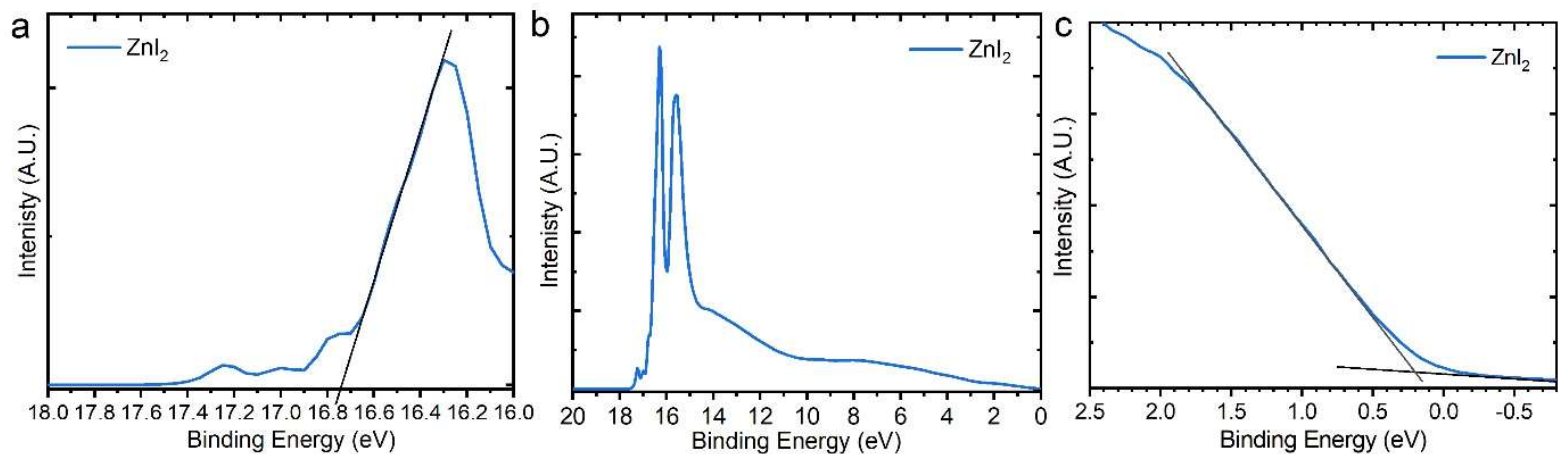

Figure S12. UPS results of the HTL with $\mathrm{ZnI}_{2}$ treatment. (a) High-binding-energy region. (b) Full UPS spectra. (c) Low-binding-energy region.
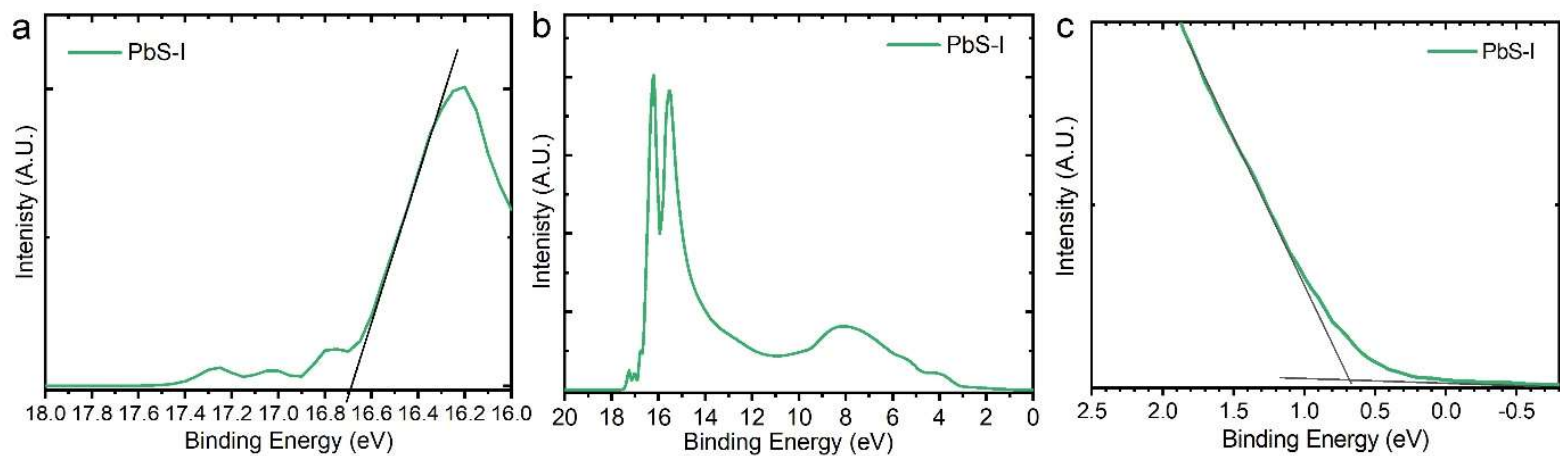

Figure S13. UPS results of the absorber layer (PbS-I). (a) High-binding-energy region. (b)

Full UPS spectra. (c) Low-binding-energy region, the cutoff energy is estimated to be $0.69 \mathrm{eV}$. 


\section{Energy level calculations:}

From Figure. 6 in the main text and Figure S8-11, for the low-binding-energy regions, the

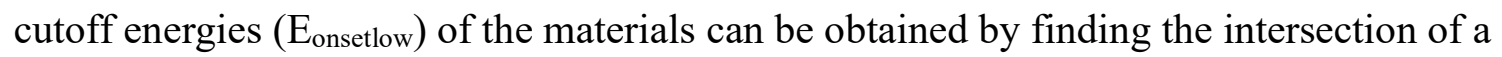
linear extrapolation of the cutoff region to the linear extrapolation of the baseline. The obtained $E_{\text {onsetlow }}$ represent the gap between the Fermi level $\left(\mathrm{E}_{\mathrm{f}}\right)$ and the valence band maximum (EVBM), and a smaller gap would indicate higher p-type characteristics. The gap between $\mathrm{E}_{\mathrm{f}}$ and the conduction band minimum $(\mathrm{CBM})$ and $\mathrm{E}_{\mathrm{f}}$ then can be estimated together with the bandgap energy of the $\mathrm{PbS}$ films, using the following equation:

$$
\left|E_{f}-E_{C B M}\right|=E_{g}-E_{\text {onsetlow }}
$$

The energy levels are then used to construct the band alignment diagram as shown in Figure $6 \mathrm{~d}$.

\section{Fermi levels:}

The position of the Fermi levels can be extracted from the cutoff of the high-binding-energy regions (Eonsethigh), together with the energy $(21.22 \mathrm{eV})$ of the $\mathrm{He}(\mathrm{I})$ source, the following formula applies:

$$
E_{f}=21.22-E_{\text {onsethigh }}
$$

The values obtained are $4.47 \mathrm{eV}$ for MPA, ZnAc and $\mathrm{ZnI}_{2}$ HTLs. For the absorber layer the energy is about $4.52 \mathrm{eV}$. All these values are highly consistent with those reported in the literatures. ${ }^{1,2}$ 


\section{References}

1. Chuang, C. H.; Brown, P. R.; Bulovic, V.; Bawendi, M. G., Improved Performance and Stability in Quantum Dot Solar Cells through Band Alignment Engineering. Nat. Mater. 2014, 13, 796-801.

2. Brown, P. R.; Kim, D.; Lunt, R. R.; Zhao, N.; Bawendi, M. G.; Grossman, J. C.; Bulovic, V., Energy Level Modification in Lead Sulfide Quantum Dot Thin Films through Ligand Exchange. ACS Nano 2014, 8, 5863-5872. 